\title{
Novel schemes for optical signal generation using laser injection locking with application to Brillouin sensing
}

\author{
L Thévenaz, S Le Floch, D Alasia and J Troger \\ EPFL Swiss Federal Institute of Technology, Nanophotonics \& Metrology Laboratory, \\ 1015 Lausanne, Switzerland \\ E-mail: Luc.Thevenaz@epfl.ch
}

Received 15 February 2004, in final form 28 March 2004

Published 19 July 2004

Online at stacks.iop.org/MST/15/1519

doi:10.1088/0957-0233/15/8/015

\begin{abstract}
Injection locking of two DFB semiconductors opens new possibilities to generate effective signals for optical sensing, in order to reach better performances. Pure wave forms can be generated with qualities exceeding those obtained using external modulators. This is illustrated through the application to the distributed Brillouin sensing that shows significant progress with respect to established techniques.
\end{abstract}

Keywords: semiconductor lasers, laser diodes, mode locking, metrological applications

\section{Introduction}

In 1665 the famous scientist Christiaan Huygens observed two clocks hanging on the wall. He noted that the pendulums of the clocks began to oscillate in phase if the clocks were brought close enough to each other, but oscillated independently when hung further apart. Huygens explained the coupling between the two clocks by mechanical vibrations transmitted through the wall. This anecdote is one of the first documented observations of the coupling of two oscillators by injection locking [1].

The injection of a weak periodic signal into a more powerful free-running oscillator may give rise to a variety of injection locking phenomena. Typically the initially freerunning oscillator (named the slave oscillator) leaves its own resonant frequency to synchronize itself with the external signal; it then gets phase locked to the injected signal from the so-called master oscillator, which thus controls the slave oscillator without being influenced by the latter. Injection locking effects may occur in virtually any kind of selfsustained oscillator, such as mechanical, electrical and laser oscillators.

In this paper we shall address an important case for applications in which two DFB semiconductor lasers are in a master-slave configuration. We first present the advantages of injection locking for optical signal processing and then show that the benefits of injection locking go beyond the simple case of a faithful replication of the master spectrum by the slave. A great variety of imaginative configurations may be defined, combining injection-locked modulated lasers, which results in the effective generation of various signals, such as pure AM, pure FM, frequency-shifted or frequency sweeping optical wave forms. Finally, we demonstrate the application of injection locking to the important case of a Brillouin fibre sensor and we show for the first time distributed measurements achieved using such a configuration.

\section{Principle}

The experimental realization of injection locking using pigtailed DFB lasers is in essence extremely simple, as shown in figure 1. Care must be taken only to properly isolate the master laser from any light injection from the slave and to make the polarization states of the two lasers matched within the slave cavity.

An important parameter is the so-called static locking range $\Delta v_{\max }$ that corresponds to the maximum difference between the free-running frequencies of the master and the slave making the frequency locking possible. In other words the frequencies of the two lasers must be tuned to lie within an interval $\Delta v_{\max }$ to enable the stable locking of the slave onto the master frequency. 


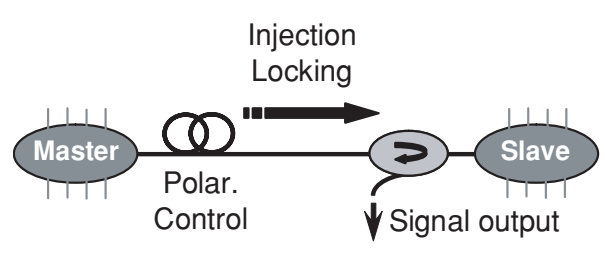

Figure 1. Basic injection-locking configuration of two DFB semiconductor lasers. The circulator isolates the master from any light of the slave and provides a lossless output.

According to standard models, the static locking range is closely related to the ratio $\rho$ between the injected master and the slave emission powers within the slave cavity [2-4]. It follows a square root relationship:

$$
\Delta v_{\max }=\frac{V_{\mathrm{g}}}{2 L} \sqrt{\left(1+\beta_{c}^{2}\right) \rho}
$$

where $V_{\mathrm{g}}$ is the group velocity, $L$ the laser cavity length and $\beta_{c}$ is the linewidth enhancement factor.

This equation shows that the higher the power injection ratio $\rho$, the broader the frequency locking range $\Delta v_{\max }$. It turns out actually in standard DFBs that for values of $\rho$ above $2 \times 10^{-5}$ unwanted relaxation oscillations are observed in the slave spectrum. And for $\rho>2 \times 10^{-4}$ the spectrum turns fully chaotic [3]. For $\rho=2 \times 10^{-5}$ the static locking range $\Delta v_{\max }$ is typically between $500 \mathrm{MHz}$ and $1 \mathrm{GHz}$. It means that the free-running frequencies of the two lasers must be initially very close to observe injection locking.

Inversely, a power injection ratio below $10^{-6}$ results in an injected power so low that it is dominated by the Rayleigh scattered slave light from the pigtail and to a static locking range comparable to the laser linewidth.

In summary a power injection ratio in the range of $10^{-5}$ is ideal to properly achieve injection locking. This approximately corresponds to the amount of power that leaks through a standard isolator in the isolating direction, so that a standard DFB isolated pigtailed module can often be used unmodified for injection locking by simply feeding the master light into the slave pigtail and then through the built-in isolator. This injection ratio condition is valid for static emission. When the lasers are modulated and the emission frequency is swept, a higher ratio is usually required for proper locking.

\section{Configurations for the generation of advanced signals}

Beyond the trivial case of injection locking when the slave laser simply perfectly replicates the master $\mathrm{CW}$ emission, it is less known that more interesting configurations can be set up to achieve more sophisticated signals. In particular the traditional drawback of mixed FM-AM modulation resulting from the direct modulation of a laser diode can be overcome to a wide extent using injection locking. Traditionally pure AM, FM and SSB modulation spectra are obtained using a single or a combination of expensive external modulators. It can be shown that the same result can be obtained with excellent performances using an injection-locking scheme with two DFB lasers. Such a solution turns out to be frequently costeffective with the further advantage of better signal purity.
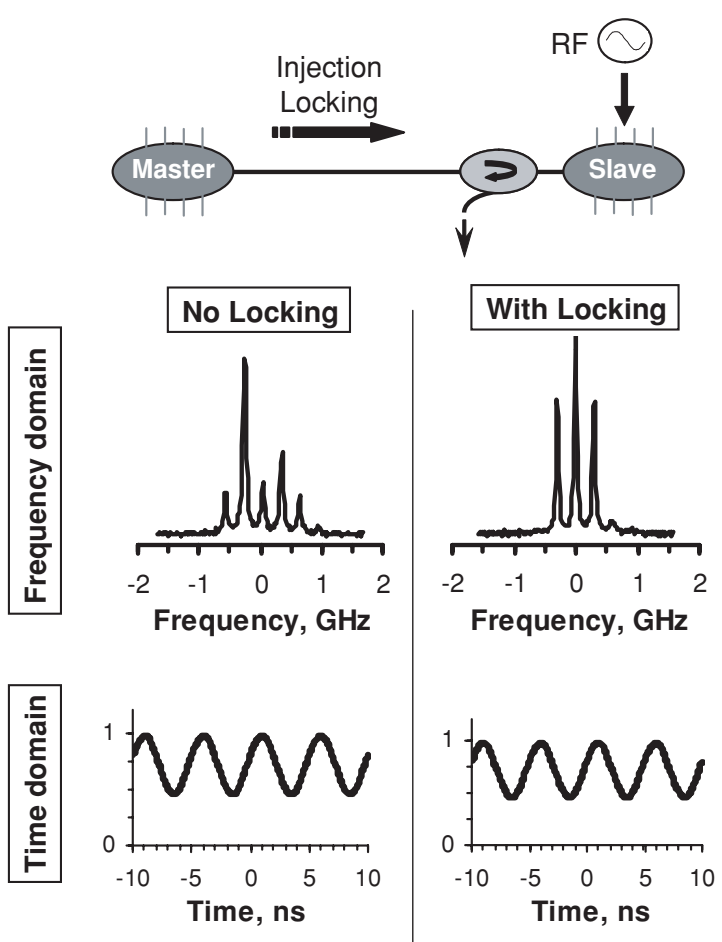

Figure 2. Injection-locking configuration for pure AM modulation. The spectrum and time-domain wave form of the slave laser are shown before and after locking to the master laser.

\subsection{Pure AM modulation}

This is achieved by operating the master laser in CW mode and modulating the current of the slave at the modulation frequency. The carrier of the slave locks on the master emission line, resulting in no frequency dithering and therefore no unwanted FM modulation, as shown in figure 2. It can be observed that the spectrum is substantially narrowed and becomes symmetrical as expected for pure AM, while the time response is unchanged with or without locking. The amount of injected power into the slave must be carefully adjusted to obtain the proper emission characteristics.

In figure 2, for a modulation frequency $f=200 \mathrm{MHz}$, the power AM modulation index $M=\Delta P / P$ remains unchanged at $M=0.2$ before and after locking, while the FM modulation index $\beta=\Delta f / f$ is changed from $\beta=6.4$ to $\beta=0.013$, demonstrating the massive reduction in frequency dithering resulting from injection locking.

\subsection{Pure FM modulation}

In this case the current of the master laser is modulated at the modulation frequency while the slave is operated in $\mathrm{CW}$ mode. The instantaneous frequency of the slave laser locks onto the instantaneous emission line of the master. The slave shows no significant change in its emission power, resulting in a pure frequency dithering, as shown in figure 3 where the slave spectrum is broad and symmetrical. While the master shows in the time domain an important AM modulation as expected from direct modulation, no AM modulation is observed in the slave emission, demonstrating pure FM modulation. Here the operating condition requires that the instantaneous frequency range lies within the locking range. 

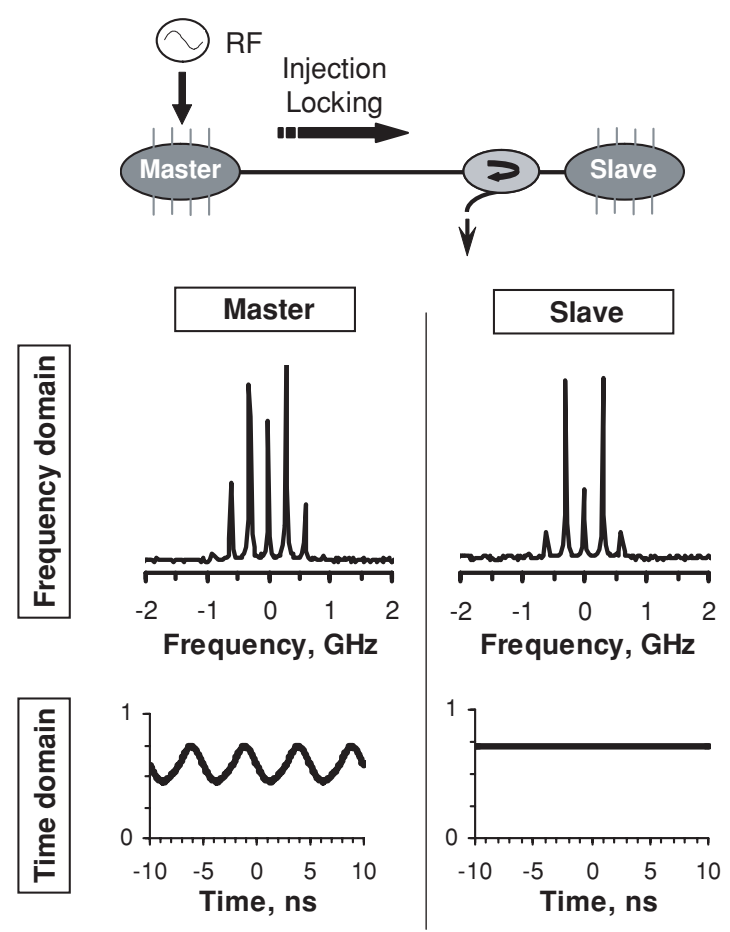

Figure 3. Injection-locking configuration for pure FM modulation. The spectra and time-domain wave forms of the master and of the slave laser are shown.

More quantitatively a FM modulation index of $\beta=2.8$ was measured, while the power AM modulation index was $M=0.41$ in the master emission and was only $M=0.009$ in the slave emission, corresponding to a $-20.5 \mathrm{~dB}$ residual $\mathrm{AM}$ modulation.

\subsection{SSB modulation (optical frequency shifting)}

This is simply achieved by modulating the current of the master laser and by locking the slave on one of the modulation sidebands. The frequency difference of the two lasers is thus perfectly stable [4-6]. The experimental configuration is identical to that shown in figure 1 , the only difference being that the modulation frequency exceeds the locking range, so that only one frequency component of the modulation spectrum matters for the injection locking. It must be pointed out that the slave laser may be locked as well on a higher order sideband, resulting in an optical frequency difference that is a multiple of the applied modulation frequency. This is particularly convenient in the microwave domain, and locking up to the fifth harmonic was successfully achieved by our team [4].

It is also possible to apply the current modulation to the slave and to obtain the same result. In this case the experimental configuration is identical to figure 2 and one of the modulation sidebands of the slave laser locks onto the master CW emission. This is formally equivalent when one considers the modulation process in the laser as a coherent coupling between two waves separated by a frequency given by the modulation. Of course an external modulator of any type can be used as well. Such a scheme may be more convenient in some experimental configurations, such as for the Brillouin fibre sensing for which this technique was successfully demonstrated, as shown in section 4.

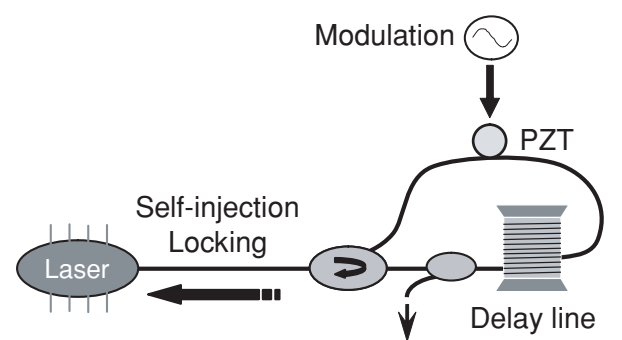

Figure 4. Injection-locking configuration for frequency sweeping generation. The slave laser is locked on its own emission, after phase modulation and a time delay resonant with the modulation.

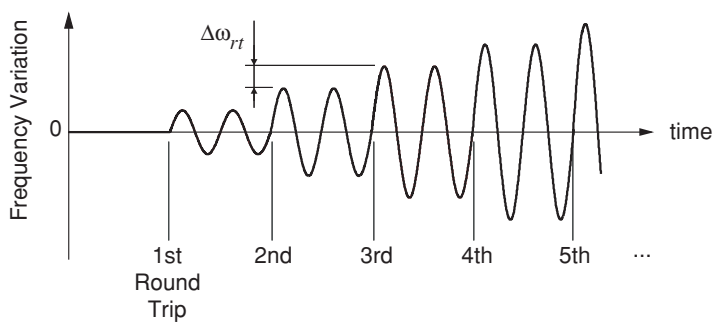

Figure 5. Instantaneous frequency variation of the fed back light at the laser input after $1,2,3, \ldots$ round trips in the fibre loop. Owing to resonant phase modulation, the frequency variations add up constructively at each round trip, $f_{m}=2 f_{0}$ in the illustrated case. 0 indicates the emission frequency of the free-running laser.

\subsection{Frequency sweeping (broadband FM)}

It is possible to generate a broadband frequency dithering with no amplitude modulation using a special scheme based on a delay line and the self-injection locking of a single laser [7]. As shown in figure 4, the laser light is first phase modulated using an external modulator that may be simply a segment of optical fibre wrapped around a piezoelectric transducer (PZT). This modulator makes the instantaneous frequency of the laser light sinusoidally modulated, at a rate given by the frequency of the electric signal applied to the PZT and an amplitude related to the phase modulation index.

This signal propagates through the fibre delay line and is then fed back into the laser cavity. The laser frequency locks on the feedback signal, showing a sinusoidal modulation of the instantaneous frequency, accordingly.

If the PZT modulation period is equal to the round trip time of the delay line, the signal is then modulated again synchronously and the instantaneous frequency shows a double sweep amplitude. This process works as well if the PZT frequency is an integer multiple of the round-trip frequency. This signal is then fed back into the laser that locks on to this doubled sweep amplitude signal. The frequency variations then add up constructively at each round trip, as shown in figure 5, until the sweep amplitude corresponds to the locking range.

When the edges of the locking range are reached the laser can no longer lock on frequencies out of the locking range and the sweeping amplitude stops growing, reaching a steady state. The obtained spectrum of the laser, shown in figure 6, is typical of a broadband FM modulation. By properly adjusting the fed-back power the frequency sweeping amplitude can be tuned, accordingly. Amplitudes up to $10 \mathrm{GHz}$ are theoretically possible with a high fed-back power in the range of $\rho=1 \%$. 


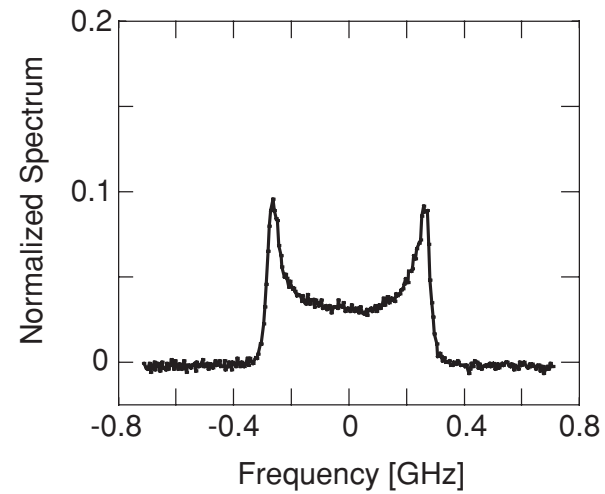

Figure 6. Power spectrum of the self-injection locked slave laser with resonant phase modulation $\left(V_{\mathrm{m}} / V_{\pi} \cong 0.27, f_{\mathrm{m}}=1.15 \mathrm{MHz}\right)$. The feedback level is $9 \times 10^{-5}$.

This is impossible to obtain without severe amplitude modulation using direct current modulation. This opens a wide field of possible applications in gas trace spectroscopy using laser diodes and in the correlation-based distributed Brillouin sensing [10].

\section{Application to distributed Brillouin sensing}

A schematic diagram of the injection locking configuration for the Brillouin sensing is shown in figure 7. A first short fibre line - the locking channel-is used to lock the frequency of the slave laser on the free running master laser by injecting a small quantity of the master light into the cavity of the slave laser. The latter is directly modulated in intensity at a frequency within the Brillouin shift range, thanks to the built-in electroabsorption modulator. This creates a small sideband that will be used for injection locking.

It must be pointed out that injection locking is performed through the built-in slave isolator in the blocking direction. The leakage power resulting from the imperfect isolation $(35 \mathrm{~dB})$ turns out to be sufficient to lock the slave laser. This feature makes possible the use of a standard commercial device with no further modification.

This configuration provides two outputs with distinct optical frequencies showing a beat note with the ideal stability given by the microwave generator. These outputs can be simply delivered in two separate fibres using couplers and circulators, as shown in figure 7 . The role of the pump can be played by either the master or the slave [8]. It just depends on which sideband the slave is locked (upper or lower). Unlike the sideband technique [9] no other idle wave is present in the set-up, except the residual modulation sideband with very low amplitude. This turns out to very significantly improve the noise characteristics of the technique.

For this reason the master laser was selected to deliver the probe signal, since it is $\mathrm{CW}$ operated with no modulation and thus contains a pure single frequency. This is the only detected signal, and the noise is consequently minimized. The power of the slave laser is boosted through an erbium-doped fibre amplifier to act as a pump in the Brillouin interaction. As for most other techniques [9], the Brillouin gain spectrum is scanned by simply changing the microwave generator frequency. Care must be taken to ensure that the locking range exceeds the frequency scanning range that extends usually over a few hundred of $\mathrm{MHz}$ and can be easily contained within the locking range using a standard injection ratio.

As described so far, the configuration makes possible the measurement of the Brillouin gain spectrum only integrated all over the entire length of the fibre placed in the measurement channel [4, 8]. But it is well known that the best advantage of Brillouin sensing is the possibility to perform distributed measurements using a localized interaction. This is traditionally achieved using a pulsed pump or probe wave for long range measurements and by retrieving the local information in the time domain.

In the injection-locking configuration, this could be simply achieved by inserting an external intensity modulator operated as a switch just before the erbium-doped fibre amplifier. But the set-up would suffer from the same drawbacks as the sideband techniques, namely the use of an expensive and poorly available electro-optic modulator.

Another solution was investigated by directly modulating the pump laser current, i.e. the slave in the injection locking set-up. Using a pulse current to turn on the laser is actually impracticable, since the transient behaviour during the turn-on process results in a delayed oscillatory response of the laser power. The duration of this transient behaviour is typically comparable to the required pulse width in a distributed Brillouin measurement (10-50 ns).

We found another solution to overcome this problem, considering that the frequency response of a fully emitting laser exceeds 1-2 GHz. The pump laser is always on and its operating current is set, so that none of the emission lines

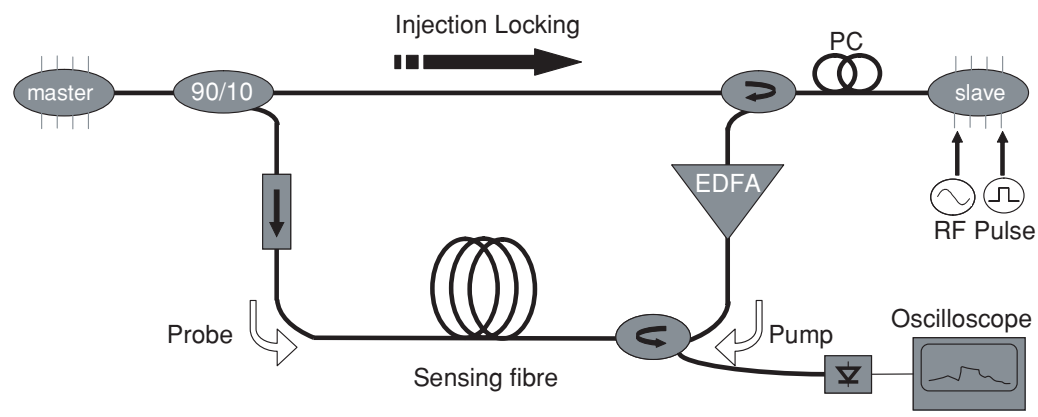

Figure 7. Schematic diagram of the injection-locking configuration for Brillouin sensing. The master injection into the slave is performed using a dedicated short fibre channel to secure a stable injection as far as amplitude and polarization are concerned. Note that the probe light is supplied by the master and the pump by the slave, since the master emission is maintained steady. 


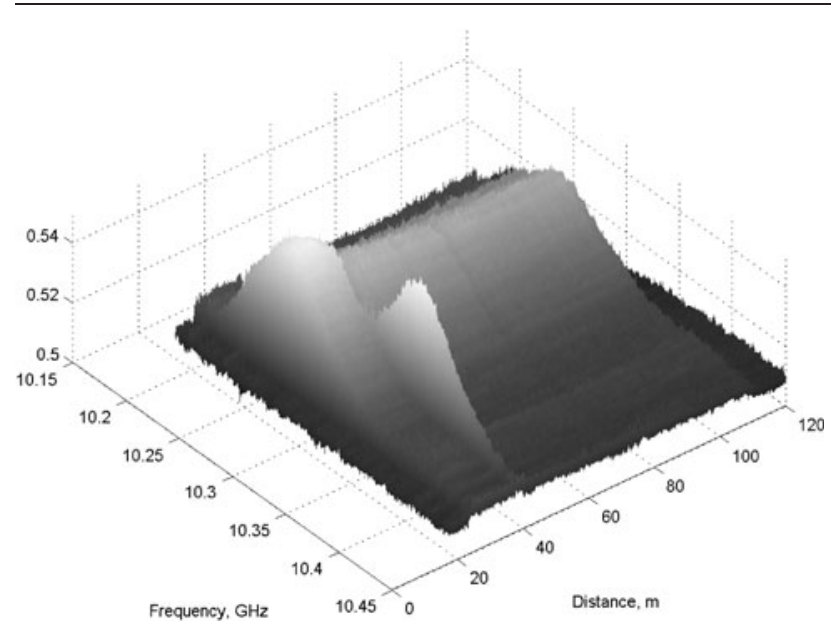

Figure 8. 3D plot of a distributed measurement of the Brillouin gain along a $90 \mathrm{~m}$ fibre obtained using a $10 \mathrm{~ns}$ pump pulse, showing the presence of a $2 \mathrm{~m}$ hot spot. The noise is remarkably low, even with a $1 \mathrm{~m}$ spatial resolution, considering the low pump power of $60 \mathrm{~mW}$ and the small amplification contrast, accordingly.

(carrier+sidebands) lie inside the locking range and the Brillouin gain spectrum. It is sufficient to lower the laser bias DC current by a couple of milliamps, corresponding to a frequency shift slightly greater than $1 \mathrm{GHz}$. The slave laser is therefore unlocked and its emission is out of the Brillouin gain spectrum, so that no interaction takes place and the observed probe signal shows no variation in the time domain.

When a current pulse is applied to the slave to move its free-running frequency into the locking range, it rapidly locks and the interaction takes place. When the pulse is stopped, the locking condition is no longer satisfied and the slave freerunning frequency is again out of the locking range and of the Brillouin spectrum, so that no interaction is possible. The Brillouin interaction is therefore possible only during the pulse duration, just as in the classical time-of-flight technique, even though the pump is always emitting and shows therefore no transient turn-on behaviour. A frequency coding in the time domain simply substitutes for the classical power coding.

The only unknown parameter is the actual time needed for the slave to lock on the master frequency. Theoretical models show that this time is significantly shorter than $1 \mathrm{~ns}$, so that we implemented this frequency-coding configuration with confidence. Figure 8 shows the raw measurement of the Brillouin amplification along a $100 \mathrm{~m}$ fibre with a $1 \mathrm{~m}$ spatial resolution, without any data processing. The presence of a $2 \mathrm{~m}$ hot spot is clearly observed, and the quality of the measured Brillouin spectra is excellent. Figure 9 shows the distribution of Brillouin frequency using different pulse widths, obtained from these spectra after processing. It clearly shows that the measurement is possible even with pulses as short as $10 \mathrm{~ns}(1 \mathrm{~m})$, and the hot spot is perfectly resolved in this case. The locking time can thus be neglected and causes no impairment for the technique.

These measurements were obtained using a pump power of $60 \mathrm{~mW}$. The quality of the measurement was extremely good and the observed noise was much lower than in the sideband technique using a Mach-Zehnder modulator [9], for which pump power over $200 \mathrm{~mW}$ is necessary to obtain the same signal-to-noise ratio. This is certainly due to the absence of co-propagating waves at other frequencies with the probe signal.

\section{Conclusion}

In this paper we present the high potentialities of injection locking applied to two DFB lasers for the generation of high quality signals for sensing applications. The obtained signals outperform in most cases those generated using external modulators at a reduced cost. The generation of pure $\mathrm{AM}, \mathrm{FM}$, frequency-shifted and frequency-swept signals is experimentally demonstrated.

These potentialities are illustrated through the application of injection locking to Brillouin distributed sensing. In

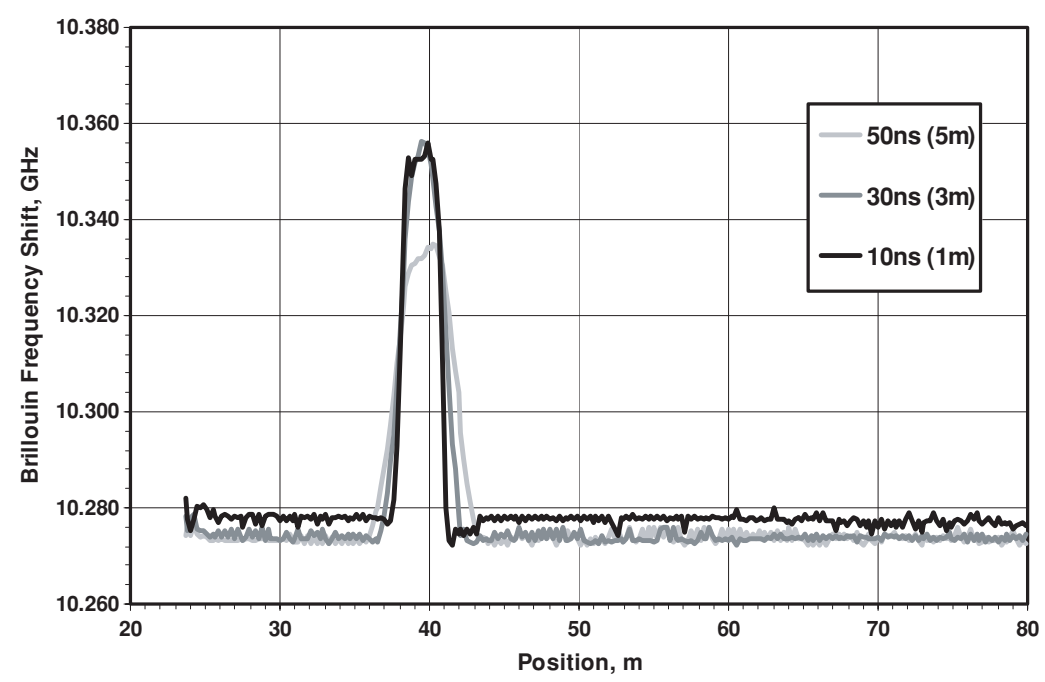

Figure 9. Distributed measurements of Brillouin frequency along a $90 \mathrm{~m}$ fibre subject to a $2 \mathrm{~m}$ hot spot, with different pulse widths. The $50 \mathrm{~ns}(5 \mathrm{~m})$ pulse does not fully resolve the hot spot, as expected. The length of the abrupt transition between the room temperature segment and the hot spot corresponds to the pulse width, showing no transient effect due to a non-instantaneous locking and demonstrating the rapidity of the process. 
particular it shows the possibility to perform a time coding of the signals using injection locking, opening the domain of time-of-flight techniques for this novel technique. The first results are definitely promising, demonstrating the flexibility of the technique and the excellence of the signals obtained with moderate equipment. This results in low noise signals that make it possible to further improve the range and the resolution of Brillouin sensing.

Other smart configurations are certainly possible using the large potentialities of injection locking and this opens a wide area for creativity in this research field.

\section{References}

[1] Siegman A E 1986 Lasers (Mill Valley, CA: University Science Books)

[2] Lang R 1982 Injection locking properties of a semiconductor laser IEEE J. Quantum Electron. 18 976-83

[3] Troger J, Nicati P-A, Thévenaz L and Robert P A 1999 Novel measurement scheme for injection-locking experiments IEEE J. Quantum Electron. 35 32-8
[4] Tröger H J 1999 Injection-locking in semi-conductor lasers PhD Thesis no 1976 EPFL, Switzerland

[5] Noel L, Marcenac D and Wake D 1996 Optical millimeter-wave generation technique with high efficiency, purity and stability Electron. Lett. 32 1997-8

[6] Jahansson L A and Seeds A J 2000 Millimeter-wave modulated optical signal generation with high spectral purity and wide-locking bandwidth using a fiber-integrated optical injection phase-lock loop Photonics Tech. Lett. 12

[7] Troger J, Thévenaz L and Robert Ph 1999 Frequency-sweep generation by resonant self-injection locking Opt. Lett. 24 $1493-5$

[8] Facchini M, Thévenaz L, Tröger J and Robert P 2002 2-laser injection locking configuration for Brillouin fibre sensors Technical Digest of 15th Optical Fiber Sensors Conf. (OFS'2002) (Portland, OR) IEEE catalogue number 02EX533 pp 313-6

[9] Niklès M, Thévenaz L and Robert Ph A 1996 Simple distributed fiber sensor based on Brillouin gain spectrum analysis Opt. Lett. 21 758-60

[10] Le Floch S and Thévenaz L 2003 Correlation-based Brillouin sensing using an injection-locking technique Technical Digest 16th Optical Fiber Sensors Conf. (OFS'2003) (Nara) (Tokyo: IEICE) paper We2-2 pp 286-9 